EPJ Web of Conferences 33, 03005 (2012)

DOI: $10.1051 /$ epjconf/20123303005

(C) Owned by the authors, published by EDP Sciences, 2012

\title{
Microemulsions as model fluids for enhanced oil recovery: dynamics adjacent to planar hydrophilic walls
}

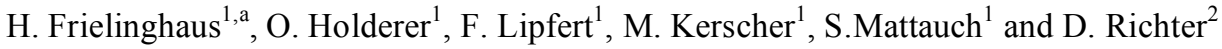 \\ ${ }^{1}$ Jülich Centre for Neutron Science, Forschungszentrum Jülich GmbH, Lichtenbergstrasse 1, 85747 \\ Garching, Germany \\ ${ }^{2}$ Institue for Complex Systems 1: Neutron Scattering, Forschungszentrum Jülich GmbH, 52425 \\ Jülich, Germany
}

\begin{abstract}
After the dynamics of microemulsions adjacent to a planar hydrophilic wall have been characterized using grazing incidence neutron spin echo spectroscopy, the model of Seifert was employed to explain the discovered acceleration for the surface near lamellar ordered membranes. Reflections of hydrodynamic waves by the wall - or the volume conservation between the membrane and the wall - explain faster relaxations and, therefore, a lubrication effect that is important for flow fields in narrow pores. The whole scenery is now spectated by using different scenarios of a bicontinuous microemulsion exposed to clay particles and of a lamellar microemulsion adjacent to a planar wall. The Seifert concept could successfully be transferred to the new problems.
\end{abstract}

\section{Introduction}

The International Energy Agency expects that in 2030 the world will still depend on oil to satisfy $29.5 \%$ of its energy demand [1]. With the primary and secondary oil production becoming increasingly difficult the development of new oil wells will be critical as well as new ways to extract oil from oil sands, extra-heavy oil and shale oil and gas. In this process the design of better or new complex fluids with tunable lubrication properties will certainly be able to make a contribution. Surfaces that the fluid is exposed to appear manifold arising from the oil hosting sand stone or from purposely added colloids and particles.

The structural changes complex fluids undergo when in contact with an interface where subject of a lot of research projects $[2,3,4,5]$ but not much is known about the dynamical behavior in the interface near regions. In [6] we stated the interesting dynamical behavior we found for an induced lamellar microemulsion adjacent to a planar hydrophilic surface namely that the induced layer (45ns) is three times faster when compared with the bicontinuous bulk structure (133ns) (see Figure 1). In addition to our conference contributions we want the present complementary experiments we conducted in order the better the understanding of what leads to this behavior.

\footnotetext{
a e-mail : h.frielinghaus@fz-juelich.de
} 


\section{Methods}

To analyze the dynamic behavior of a bulk microemulsion neutron spin echo spectroscopy is the preferred method and has been successfully applied for many years [7,8,9]. In order to gain information about the near surface dynamics with the benefit of depth resolution due to evanescent scattering we ported the grazing incidence techniques used for GISANS $[2,10]$ and applied them to neutron spin echo spectroscopy.

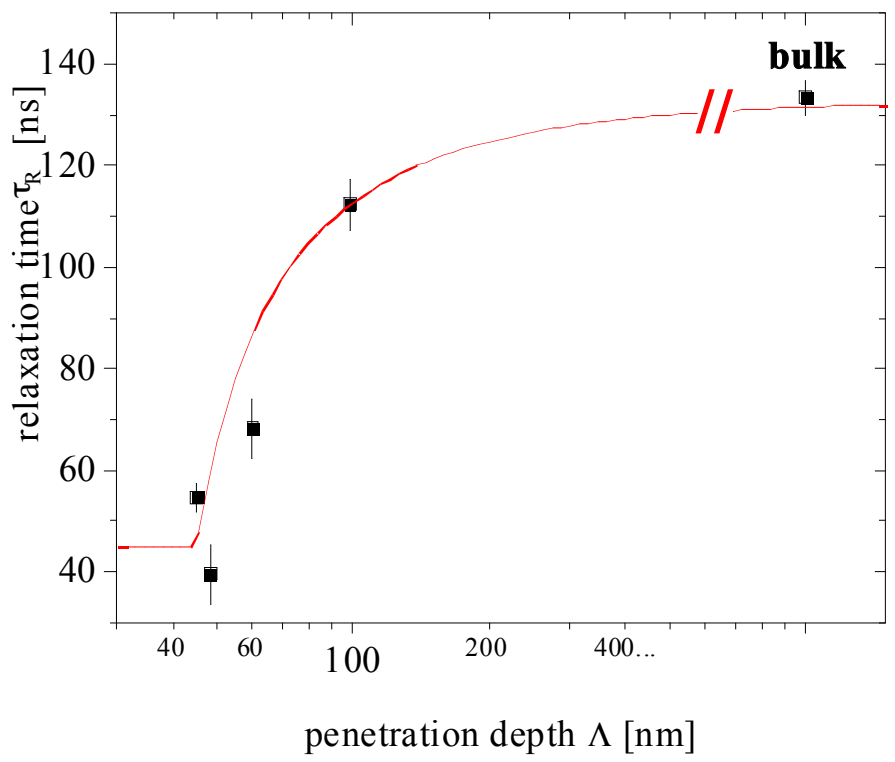

Fig. 1 relaxation times of multiple GINSES measurements vs. penetration depth into the sample.

\subsection{GISANS}

In grazing incidence small angle neutron scattering, the neutron beam hits the surface of a Si-block below the critical angle of total reflection determined by the contrast between the silicon and the liquid. Because the primary beam is totally reflected by the interface only an evanescent (tunneling) wave penetrates the sample, giving rise to the scattering signal. How far the evanescent wave penetrates into the sample is determined by the contrast and on the incident angle of the incoming beam. A variation of these two parameters thus allows us to measure scattering from regions closer or farther away from the interface.

\subsection{GINSES}

Grazing incidence neutron spin echo spectroscopy is a new technique that makes it possible to analyze the near surface dynamics of liquids adjacent to a planar silicon block that can be chemically altered to present the liquid to different environments. The grazing incidence nature of the experimental setup makes it possible to gain dynamic information about the system near the interface at different distances to it due to the penetration into the sample being only an evanescent wave. The depth resolution behaves in the same way as for GISANS experiments. If the angle of the incoming neutron beam is kept below the critical angle of total reflection, then changing the incoming angle varies the penetration depth. 


\section{Results}

Three complementary experiments were conducted on symmetric microemulsions containing equal amounts of water $\left(\mathrm{H}_{2} \mathrm{O} / \mathrm{D}_{2} \mathrm{O}\right)$ and oil (h-decan/d-decan) in order to better the understanding of the near-surface dynamics of microemulsions. All experiments were performed at the FRM II, the NSE and GINSES measurements at J-NSE and the GISANS measurements at MARIA.

\subsection{Brief review on microemulsion adjacent to a planar hydrophilic surface}

The main result of these GINSES experiments is summarized in Figure 1 [6]. While at low penetration depths we highlighted the near surface lamellar structure of the microemulsion. This structure shows typical relaxation times of $45 \mathrm{~ns}$, which is three times faster than the bulk bicontinuous microemulsion (133ns). This acceleration was interpreted on the basis of the Seifert model $[11,12]$. Close to the surface long wavelength modes exist which are much faster than the corresponding microemulsion modes (Figure 2), which is explained by the hydrodynamic waves being reflected at the wall or simply by the conservation law of the volume between a membrane and a wall. The faster relaxation times have to be seen in context with the lubrication effect. Parallel membranes allow for a faster sliding along the wall direction, which facilitates the pumping of microemulsions in narrow pores.

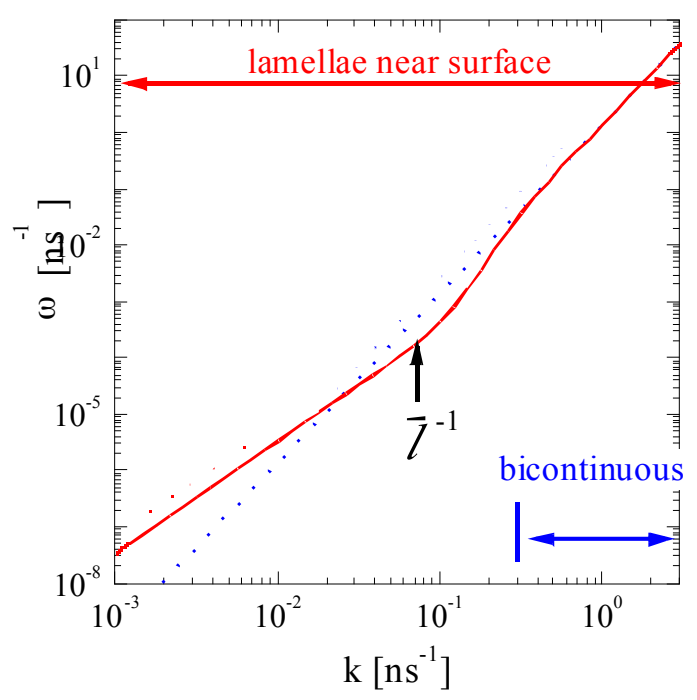

Fig. 2 The Seifert dispersion relation for a membrane adjacent to a planar wall (or neighboring membrane) (red) compared to the dispersion relation of a freestanding membrane in the simple Zilman-Granek model (blue). In either case, hydrodynamic effects are taken into account. In the short wavelength limit the membrane only interacts with itself, while in the long wavelength limit reflections at the wall come into play, or simply the volume conservation between the wall and the membrane becomes important. For bicontinuous microemulsions and non-aligned lamellar microemulsions, the patch size $\xi$ comes into play as a long wavelength cutoff $\mathrm{k}_{\min }=$ $\pi / \xi$.

\subsection{Microemulsions and clay}

Clays are used in drilling fluids for their shear thinning effect and thus might be found as compounds in the oil field. The used clay consists of thin disk-like platelets with a thickness of about $1 \mathrm{~nm}$ and a 
diameter of $80 \mathrm{~nm}$. The idea of this setup is that the clay is supposed to provide a microscopically flat hydrophilic interface, similar to the single flat silicon block in the grazing incidence experiments. Dispersed in a bicontinuous microemulsion, we expected that a lamellar ordering would be induced at the disk interfaces. In this scenario increasing the clay concentration, would mean increasing the fraction of lamellar to bicontinuous microemulsion from a completely bicontinuous to a completely lamellar structure. The microemulsion had to be matched to the scattering length density (SLD) of the clay particles. A contrast matching study of the clay particles in water was performed at the SANS instrument KWS-1 at the FRM II to find the matching composition which was determined to a $\mathrm{H}_{2} \mathrm{O}: \mathrm{D}_{2} \mathrm{O}$ ratio of $31: 69$. The surfactant concentration was $17 \%$ of the whole sample with equal amounts of $\mathrm{D}_{2} \mathrm{O}$ and h-decan/d-decan (45:55). The contrast-matched samples were measured as a series with clay concentrations of $0 \%, 0.05 \%, 0.1 \%, 0.15 \%$ and $0.2 \%$. The intermediate scattering function at $\mathrm{q}=0.8 \mathrm{~nm}^{-1}$ is shown in Figure 3 . The bottom line is that the relaxation rate was not affected by the presence of the clay platelets.

This might be due to two reasons: either, the clay does not induce the lamellar ordering at all, or it has no effect on the relaxation rate. In our approach we interpreted the change of the relaxation rate close to an interface in terms of a modified dispersion relation according to the Seifert-theory in the Zilman-Granek expression of an undulating membrane patch in a viscous medium (Figure 2). The dispersion relation depends on the undulation mode wave vector $\mathrm{k}$ by $\mathrm{k}^{2}$ and not by $\mathrm{k}^{3}$ as in the bulk. It turns out, that the long wavelength undulation modes are mainly affected by the transition from $\mathrm{k}^{3}$ to $\mathrm{k}^{2}$ behavior. The transition takes place at $\mathrm{q} \approx 0.01 \mathrm{~nm}^{-1}$, i.e. at real space distances of about $600 \mathrm{~nm}$, so on length scales much larger than the platelet size. Thus, the actual long wavelength cutoffs in our case are

$\mathrm{k}_{\min }=2 \pi / 80 \mathrm{~nm}=0.08 \mathrm{~nm}^{-1} \quad$ and $\quad \mathrm{k}=2 \pi /(2 \xi)=2 \pi / 20 \mathrm{~nm}=0.3 \mathrm{~nm}^{-1}$

for the lamellar microemulsion at the clay particle and the corresponding bicontinuous microemulsion. It is therefore easily understandable that for the clay containing microemulsions, the affected long wavelength undulation modes were suppressed by the short extend of the lamellar phase on the platelet. Even more so, Figure 4 shows that independent of the probed length scale the relaxation is the same for each of the $0 \%$ and the $0.2 \%$ clay samples.

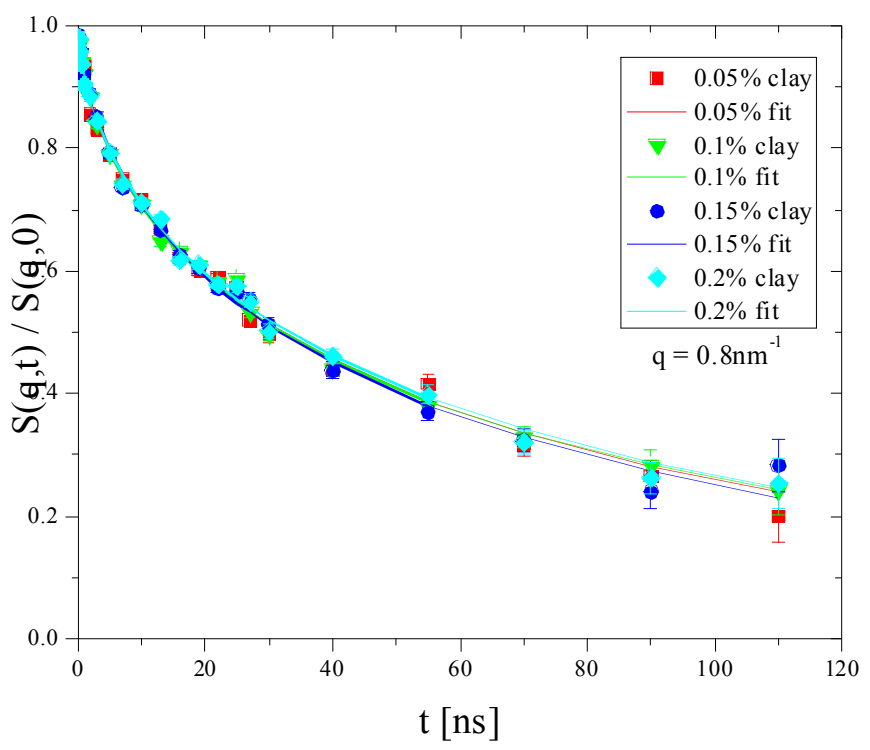

Fig. 3 Bicontinuous microemulsion with clay particles with a clay-concentration of $0.05-0.2 \%$ 


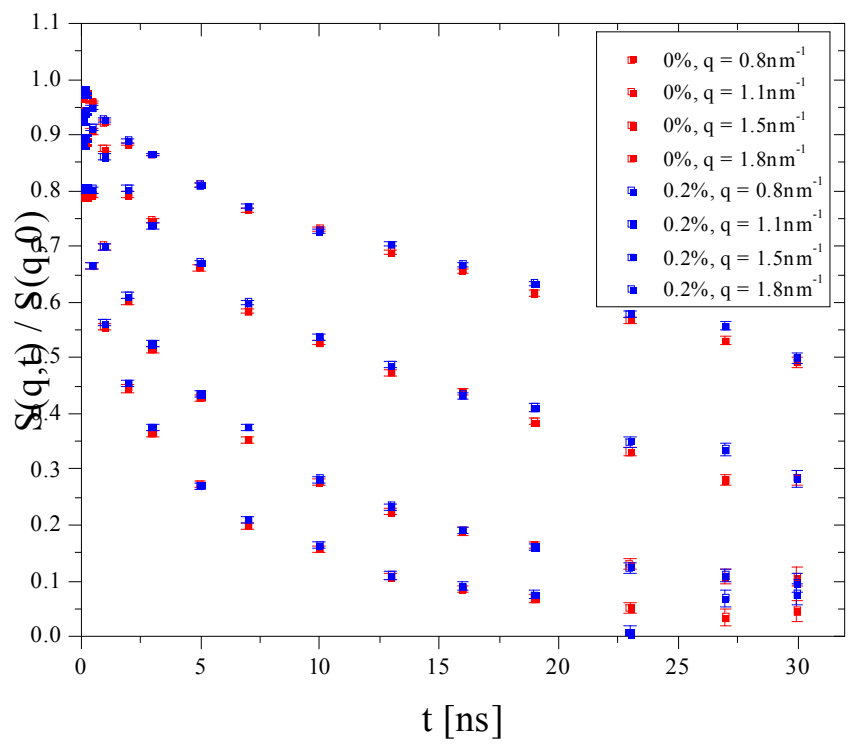

Fig. 4 Intermediate scattering function for $\mathrm{q}=0.8 \mathrm{~nm}^{-1}, 1.1 \mathrm{~nm}^{-1}, 1.5 \mathrm{~nm}^{-1}, 1.8 \mathrm{~nm}^{-1}$ each for $0 \%$ and $0.2 \%$ clay.

\subsection{Additional experiments on the faster interface dynamics}

A distinct decrease of the relaxation time has been observed at different penetration depths between the near-surface lamellar structure and the bicontinuous phase with GINSES [6]. The question arose whether this acceleration is related to an inherent property of the lamellar phase or is induced by the confinement due to the interface.

\subsubsection{High surfactant NSE}

The question was addressed with the use of a sample with a much higher surfactant concentration of $32 \%$ compared to $17 \%$, where a transition between the bicontinuous bulk and the lamellar bulk phase can be induced by changing the sample temperature. The domain sizes are much smaller than in the samples with $17 \%$ surfactant concentration used in the previous GINSES studies. The decrease in domain size shifts the correlation peak to a higher value of $q=0.64 \mathrm{~nm}^{-1}$ with a distinct second order peak at $\mathrm{q}=1.29 \mathrm{~nm}^{-1}$ (Figure 6 inlay). While for the $17 \%$ microemulsion the correlation peak was located at $\mathrm{q}=0.32 \mathrm{~nm}^{-1}$ and the GINSES experiments were performed at $\mathrm{q}=0.8 \mathrm{~nm}^{-1}$, these experiments were conducted at $\mathrm{q}=1.1 \mathrm{~nm}^{-1}$ in order to compensate the shifted correlation peak. Nevertheless it still has a slightly higher relaxation time than the $17 \%$ microemulsions at $\mathrm{q}=$ $0.8 \mathrm{~nm}^{-1}$, which is due to the proximity to the correlation peak and $\mathrm{q}=1.1 \mathrm{~nm}^{-1}$ being in the valley between the first and second order correlation peaks.

In Figure 5, the relaxation time is plotted as a function of temperature. Across the transition region, no change of the relaxation time was observed. The bicontinuous phase seems to have a slightly smaller relaxation time than the lamellar one, which we attribute to a slight shift of the correlation peak towards higher q for the lamellar phase, which moves the observed q value closer to the correlation peak. Acceleration due to the presence of the lamellar phase could not be observed. We therefore conclude that the accelerated relaxation close to the Si interface in GINSES experiments is indeed a surface induced effect. The here considered bulk lamellar microemulsion is a 'powder' sample with many small domains of lamellar order. The typical membrane patch size in the 
bicontinuous microemulsion and the bulk lamellar microemulsion do not differ, and so long wavelength modes are inhibited in either case.

\subsubsection{High surfactant GINSES}

The inlay in Figure 6 shows a GISANS picture which was recorded with an incoming angle slightly over the critical angle of total reflection. In this case the scattering is not due to evanescent wave scattering, which means that the penetration depth becomes infinite, meaning the whole sample is illuminated. The ring like scattering around $\mathrm{q}=0.64 \mathrm{~nm}^{-1}$ and less around $\mathrm{q}=1.29 \mathrm{~nm}^{-1}$ comes from the microemulsion farther away from the interface where the lamellar ordering seems to be less distinct. In complementary measurements with a short penetration depths we saw only the peak at $q$ $=0.64 \mathrm{~nm}^{-1}$ telling us that close to the interface the ordering is in deed lamellar.

Figure 6 shows the corresponding intermediate scattering function for the bulk microemulsion and for the microemulsion under grazing incidence with a penetration depth of $50 \mathrm{~nm}$. We found the relaxation times to be almost identical in the bulk and near the surface with the surface relaxation time being slightly higher than the one of the bulk.

The in [6] presented behavior seems to stem from the fact that the normal repetition unit of oilsurfactant-water-surfactant is reduced to half its width for the first layer at the interface which leads to the increased dynamics. In the case of the microemulsion already being in a completely lamellar state and having much smaller repetition units due to the increased surfactant concentration, the contact to the interface has no influence. So different neighbors of undulating membranes do not influence the relaxation spectrum at high surfactant concentrations. A flat neighboring membrane and the solid surface have similar effects in this case.

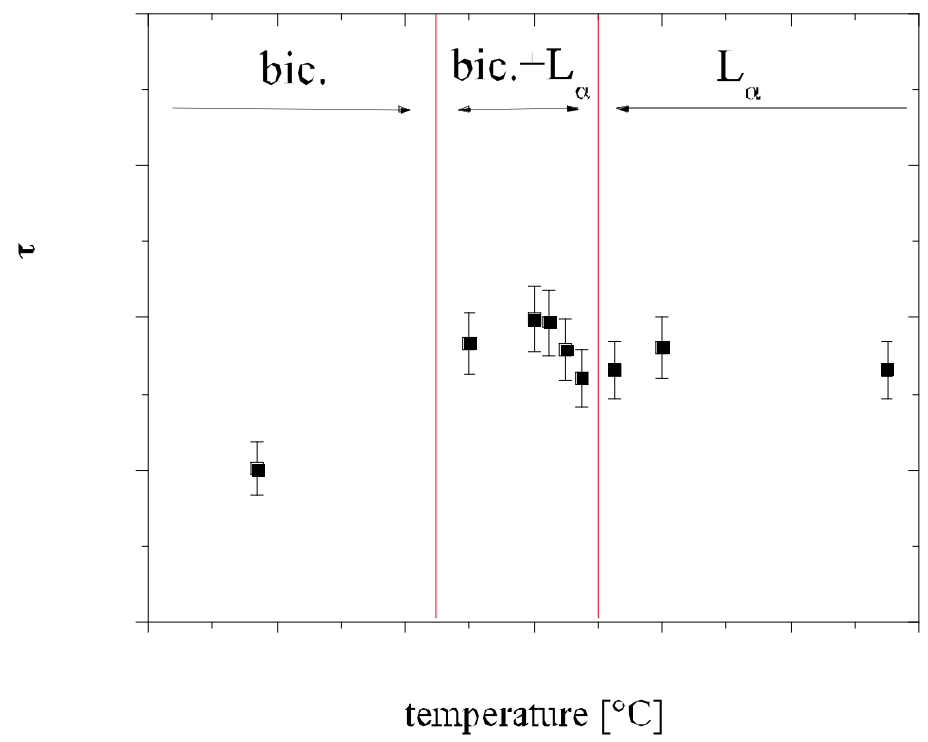

Fig. 5 Bulk NSE experiments at $\mathrm{q}=1.1 \mathrm{~nm}^{-1}$, at a series of temperatures. The sample is in the bicontinuous phase below $20.5^{\circ} \mathrm{C}$, in the lamellar phase above $23{ }^{\circ} \mathrm{C}$, with a coexistence region in between. 


\section{Conclusions}

The experiments on clay containing microemulsions suggest that it is indeed the long wavelength undulation modes that make the difference and are responsible for the faster near-interface relaxation observed in bicontinuous microemulsions. We saw two cases of lamellar microemulsions. The nonaligned lamellar microemulsion has a powder structure and does not differ dynamically strongly from the bicontinuous microemulsion. At high surfactant concentrations, the exact neighbor being a solid surface or another flat membrane does not cause any difference.

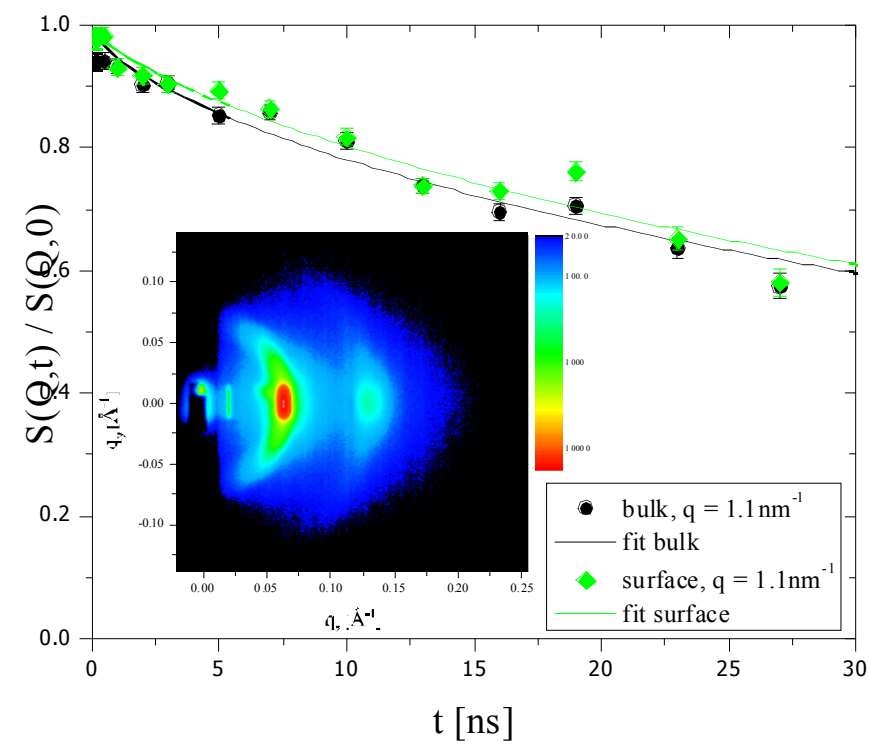

Fig. 6 Lamellar microemulsion, in the bulk and close to the interface by a GINSES experiment, inlay: GISANS of completely lamellar microemulsion, first and second order lamellar peak at $\mathrm{q}_{\mathrm{z}}=0.64 \mathrm{~nm}^{-1}$ and $\mathrm{q}_{\mathrm{z}}=1.29 \mathrm{~nm}^{-1}$, above the critical angle of total reflection at $\lambda=0.45 \mathrm{~nm}$

\section{References}

1. International Energy Agency: Key World Energy Statistics. print, 9, rue de la Fédéartion, 75739 Paris Cedex 15, 2010.

2. Kerscher, M., Busch, P., Mattauch, S., Frielinghaus, H., Richter, D., Belushkin, M., Gompper, G., Phys. Rev. E 83, 030401 (2011)

3. Zhou, X.-L., Lee, L.-T., Chen, S.-H., Strey, R., Phys. Rev. A 46, 6479 (1992)

4. Lang, P., J. Phys.: Condens. Matter 16, R699 (2004)

5. Fragneto-Cusani, G., J. Phys.: Condens. Matter 13, 4973 (2001)

6. Frielinghaus, H., Kerscher, M., Holderer, O., Monkenbusch, M., and Richter, D., Physical Review E, (2012)

7. Mezei, F. (ed.), Springer 128, (1980).

8. Mihailescu, M., Monkenbusch, M., Endo, H., Allgaier, J., Gompper, G., Stellbrink, J., Richter, D., Appl. Phys. A: Mater. 74, 414 (2002).

9. Monkenbusch, M., Holderer, O., Frielinghaus, H., Byelov, D., Allgaier, J., \& Richter, D., J. Phys.: Condens. Matter 17, 2903 (2005)

10. Müller Buschbaum, P., Gutmann, J., Cubitt, R., \& Petry, W., Physica B 350, 207 (2004)

11. U. Seifert, Phys. Rev. E 49, 3124 (1994)

12. M. Kraus, U. Seifert, J. Phys. II France 4, 1117 (1994) 